\title{
Aberrant expression for microRNA is potential crucial factors of haemorrhoid
}

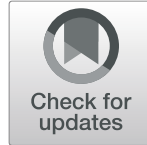

Chengkun Song ${ }^{1 \dagger}$, Haikun Zhou ${ }^{1 \dagger}$, Hong Lu ${ }^{1 \dagger}$, Chunsheng Luo ${ }^{1 \dagger}$, Chen Wang ${ }^{1}$, Qingming Wang ${ }^{1}$, Yunhua Peng ${ }^{1}$, Yaojie $\mathrm{Xin}^{2}, \mathrm{Te}_{\mathrm{Liu}}^{3,4^{*}}$ and Wei Yang ${ }^{*^{*}}$

\begin{abstract}
Background: Haemorrhoids occur commonly and frequently in the human digestive system. There are diverse causes of haemorrhoids and their in-depth pathogenesis is still currently unclear.

Methods: In this study, we explored haemorrhoids from an epigenetics perspective by employing RNA-Seq for comprehensive and in-depth analysis of the differences in microRNA (miRNA) transcripts between haemorrhoidal tissue and normal tissue in 48 patients with Grade II and above haemorrhoids.

Results: The results showed that 9 miRNAs were significantly upregulated (ratio $>3.5$ and $P$-value $<0.01$ ) and 16 miRNAs were significantly downregulated (ratio $>0.6$ and $P$-value $<0.01$ ) in haemorrhoid tissue. Subsequently, target gene prediction results showed that there were 184 potential target genes of significantly upregulated miRNAs (common to both TargetScan7.1 and MirdbV5 databases) and there were 372 potential target genes of significantly downregulated miRNAs. Gene ontology analysis results showed that the target genes of differentially expressed miRNAs in haemorrhoids are involved in regulating "cell composition" and "protein binding". Lastly, KEGG search found that the differentially expressed miRNAs that are associated with the occurrence of haemorrhoids mainly regulate the activity of endocytosis and the synaptic vesicle cycle.

Conclusions: In summary, the results of high-throughput RNA-Seq screening suggested that the occurrence of haemorrhoids may be intimately associated with aberrant miRNA transcription, resulting in aberrant target gene expression and an imbalance in certain signal transduction pathways.
\end{abstract}

Keywords: Haemorrhoids, microRNA, RNA-Seq, Signal transduction pathways

\section{Introduction}

Piles, also known as haemorrhoids, is a common disease of the anus. This condition can occur at any age but its incidence increases with age $[1,2]$. The main presentation of haemorrhoids is haematochezia, characteristics of

\footnotetext{
* Correspondence: 0721160004@mail.tongji.edu.cn; 13917198396@163.com ${ }^{+}$Chengkun Song, Haikun Zhou, Hong Lu and Chunsheng Luo these authors contributed equally to this work and shared the first authorship.

${ }^{3}$ Shanghai Geriatric Institute of Chinese Medicine, Shanghai University of Traditional Chinese Medicine, 365 South Xiangyang Road, Shanghai 200031 P.R. China

'Department of Anorectal Surgery, Shuguang Hospital, Shanghai University of Traditional Chinese Medicine, 528 Zhangheng Road, Shanghai 201203, P.R. China

Full list of author information is available at the end of the article
}

which include painless, intermittent, fresh blood after bowel movements, dripping during bowel movements or blood on toilet paper, constipation, or aggravation after alcohol consumption or consumption of irritant foods. The location where haemorrhoids occur can be classified as internal haemorrhoids, external haemorrhoids, or mixed haemorrhoids [1-3]. The aetiology of haemorrhoids is extremely complex, yet their incidence is extremely high. Personal hygiene, lifestyle habits, dietary habits, physical differences, genetic factors, and immune factors can all induce the formation of haemorrhoids [4-6]. However, there is a lack of in-depth and

(c) The Author(s). 2020 Open Access This article is licensed under a Creative Commons Attribution 4.0 International License, which permits use, sharing, adaptation, distribution and reproduction in any medium or format, as long as you give appropriate credit to the original author(s) and the source, provide a link to the Creative Commons licence, and indicate if changes were made. The images or other third party material in this article are included in the article's Creative Commons licence, unless indicated otherwise in a credit line to the material. If material is not included in the article's Creative Commons licence and your intended use is not permitted by statutory regulation or exceeds the permitted use, you will need to obtain permission directly from the copyright holder. To view a copy of this licence, visit http://creativecommons.org/licenses/by/4.0/ The Creative Commons Public Domain Dedication waiver (http://creativecommons.org/publicdomain/zero/1.0/) applies to the data made available in this article, unless otherwise stated in a credit line to the data. 
comprehensive research on the role of epigenetic regulation in the development of haemorrhoids.

Epigenetics research has found that a class of noncoding RNAs are present in the cytoplasm with lengths of 21-23 nt. These non-coding RNAs are highly conserved during species evolution and in sequence homology. However, there are no open reading frames (ORF) in these sequences so they do not encode any polypeptides. These signs suggest that they play important physiological roles in cells and biological organisms [7-12]. In our previous study, we found that DLK1-DIO3 Imprinted Cluster miRNA expression differed between clinical haemorrhoid samples and normal perianal tissues. An in-depth study found that miRNA-412-5p regulates the epigenetic mechanism of Exportin1 (Xpo1) and down stream p53/p66Shc/ p16 pathway on vascular endothelial cell proliferation and haemorrhoid formation [13]. Therefore, the microRNA regulation for the occurrence of hemorrhoids is very important. However, there have been no in-depth and comprehensive reports on whether miRNAs play regulatory roles during the occurrence and development of haemorrhoids. Therefore, this paper uses human haemorrhoidal tissue as study subjects. Transcriptomics methods (RNASeq) was employed as a study method with healthy human tissue as controls for comprehensive analysis of differentially expressed miRNA transcript population. In addition, bioinformatics was used to examine the potential target genes of the aforementioned miRNAs and their functions.

\section{Materials and methods}

\section{Collection and grouping of tissue samples}

In this study, haemorrhoidal samples were collected from 48 male patients who underwent haemorrhoid surgery in the Department of Proctology in Shanghai Shuguang Hospital from September 2017 to April 2018. The ages of all patients were between 28 to 50 years $(40 \pm 8)$, and the clinical stage of haemorrhoids was Grade III or IV. The study protocol was approved by the Regional Ethics Committee of Shuguang Hospital, Shanghai University of Traditional Chinese Medicine (Permission No.: 201701.4), in accordance with the 2008 Helsinki declaration. Written informed consent was obtained from every patient and control.

\section{RNA extraction}

RNA was extracted using the RNAprep pure Tissue Kit (TIANGEN Biotech (Beijing) Co., Ltd), following the manufacturer's instructions. Briefly, $20 \mathrm{mg}$ of human tissue samples were taken and $800 \mu \mathrm{l}$ of lysis buffer was added before homogenisation. The supernatant was obtained and $200 \mu \mathrm{l}$ of chloroform was added and mixed evenly by inverting. Following that, the samples were centrifuged at $13,400 \times g$ for $15 \mathrm{~min}$ at $4{ }^{\circ} \mathrm{C}$ and the supernatant was collected. Two volumes of absolute ethanol were added to the supernatant and mixed evenly. Following that, the samples were centrifuged at $13,400 \times g$ for $30 \mathrm{~min}$ at $4{ }^{\circ} \mathrm{C}$. Five hundred microlitersof $75 \%$ ethanol was added to the RNA precipitate for resuspension before centrifugation at $13,400 \times g$ for $5 \mathrm{~min}$ at $4{ }^{\circ} \mathrm{C}$. Excess liquid was removed and $300 \mu \mathrm{L}$ DEPC water was added to solubilise the precipitate. RNA solution $(1 \mu \mathrm{l})$ was used to measure the $\mathrm{OD}_{260} / \mathrm{OD}_{280}$ (generally controlled within 1.8-2.0) to determine the purity and total concentration of RNA.

\section{Reverse transcription of RNA into cDNA}

Reverse transcription was carried out using the miRcute miRNA First-strand cDNA (TIANGEN Biotech (Beijing) Co., Ltd) kit, following the manufacturer's instructions. Briefly, $20 \mu \mathrm{l}(100 \mathrm{ng} / \mu \mathrm{l})$ of total RNA, $25 \mu \mathrm{l}$ of $2 \times$ miRNA RT Reaction Buffer, $4 \mu$ of $1 \times$ miRNA RT Enzyme Mix and $6 \mu \mathrm{l}$ of RNAse-free deionised water were fully mixed. The following reaction was carried out on a PCR machine: $42{ }^{\circ} \mathrm{C}$ for $60 \mathrm{~min}$ for the addition of a poly (A) tail to miRNA and reverse transcription followed by $95^{\circ} \mathrm{C}$ for $3 \mathrm{~min}$ for enzyme inactivation.

\section{MiRNA qPCR}

qPCR was performed using the miRcute miRNA qPCR Detection (TIANGEN Biotech (Beijing) Co., Ltd) kit, following the manufacturer's instructions. Briefly, the following reaction system was set up: $10 \mu \mathrm{l}$ of $2 \times$ miRcute Plus miRNA Premix (with SYBR), $1 \mu \mathrm{l}(10 \mu \mathrm{M})$ each of $1 \times$ Forward Primer and Reverse Primer, $4 \mu \mathrm{l}$ of miRNA first strand cDNA, and $4 \mu \mathrm{l}$ of deionised water. The following reaction was carried out on areal-time fluorescence quantitative PCR machine: $95^{\circ} \mathrm{C}$ for $15 \mathrm{~min}, 94{ }^{\circ} \mathrm{C}$ for $20 \mathrm{~s}, 60^{\circ} \mathrm{C}$ for $34 \mathrm{~s}$, and the fluorescence value was read. The aforementioned reaction was repeated for 40 cycles. Table 1 shows the qPCR primers.

\section{Haematoxylin-eosin (H\&E) staining}

Tissue samples were fixed with $4 \%$ paraformaldehyde followed by dehydration and paraffin embedding. Fourmicronthick sections were cut from the paraffin blocks and pasted on glass slides. Subsequently, xylene was used for clearing followed by dehydration with an ethanol gradient (100, 90, 80\%) dehydration. Haematoxylin staining was carried out at room temperature for $5 \mathrm{~min}$ before differentiation using $1 \%$ hydrochloric acid in ethanol, bluing with ammonia water for $1 \mathrm{~min}$, and rinsing with distilled water for $5 \mathrm{~min}$. Subsequently, eosin staining at room temperature for 2 min was carried out followed by rinsing with distilled water for $2 \mathrm{~min}$. This was followed by ethanol gradient $(75,80,95,100 \%)$ decolorisation. Xylene was used for clearing for $2 \mathrm{~min}$. Finally, neutral resin was used for mounting. 
Table $1 \mathrm{qPCR}$ primers

\begin{tabular}{|c|c|}
\hline Gene Name & $\begin{array}{l}\text { Forward }(F) \text { and reverse }(R) \text { primers } \\
\left(5^{\prime} \rightarrow 3^{\prime}\right)\end{array}$ \\
\hline hsa-miR-375 & $\begin{array}{l}\text { F: TTGTTCGTTCGGCTCGCGTGA } \\
\text { R: GCTGTCAACGATACGCTACCTA }\end{array}$ \\
\hline hsa-miR-215-5p & $\begin{array}{l}\text { F: ATGACCTATGAATTGACAGAC } \\
\text { R: GCTGTCAACGATACGCTACCTA }\end{array}$ \\
\hline hsa-miR-192-5p & $\begin{array}{l}\text { F: CTGACCTATGAATTGACAGCC } \\
\text { R: GCTGTCAACGATACGCTACCTA }\end{array}$ \\
\hline hsa-miR-143-3p & $\begin{array}{l}\text { F: TGAGATGAAGCACTGTAGCTC } \\
\text { R: GCTGTCAACGATACGCTACCTA }\end{array}$ \\
\hline hsa-miR-187-3p & $\begin{array}{l}\text { F: TCGTGTCTTGTGTTGCAGCCGG } \\
\text { R: GCTGTCAACGATACGCTACCTA }\end{array}$ \\
\hline hsa-miR-194-5p & $\begin{array}{l}\text { F: TGTAACAGCAACTCCATGTGGA } \\
\text { R: GCTGTCAACGATACGCTACCTA }\end{array}$ \\
\hline hsa-miR-194-5p & $\begin{array}{l}\text { F: TGTAACAGCAACTCCATGTGGA } \\
\text { R: GCTGTCAACGATACGCTACCTA }\end{array}$ \\
\hline hsa-miR-145-5p & $\begin{array}{l}\text { F: GTCCAGTTTCCCAGGAATCCCT } \\
\text { R: GCTGTCAACGATACGCTACCTA }\end{array}$ \\
\hline hsa-miR-490-3p & $\begin{array}{l}\text { F: CAACCTGGAGGACTCCATGCTG } \\
\text { R: GCTGTCAACGATACGCTACCTA }\end{array}$ \\
\hline hsa-miR-145-3p & $\begin{array}{l}\text { F: GGATTCCTGGAAATACTGTTCT } \\
\text { R: GCTGTCAACGATACGCTACCTA }\end{array}$ \\
\hline hsa-miR-139-3p & $\begin{array}{l}\text { F: TGGAGACGCGGCCCTGTTGGAGT } \\
\text { R: GCTGTCAACGATACGCTACCTA }\end{array}$ \\
\hline hsa-miR-376b-3p & $\begin{array}{l}\text { F: ATCATAGAGGAAAATCCATGTT } \\
\text { R: GCTGTCAACGATACGCTACCTA }\end{array}$ \\
\hline hsa-miR-34a-5p & $\begin{array}{l}\text { F: TGGCAGTGTCTTAGCTGGTTGT } \\
\text { R: GCTGTCAACGATACGCTACCTA }\end{array}$ \\
\hline hsa-miR-379-3p & $\begin{array}{l}\text { F: TATGTAACATGGTCCACTAACT } \\
\text { R: GCTGTCAACGATACGCTACCTA }\end{array}$ \\
\hline hsa-miR-190b & $\begin{array}{l}\text { F: TGATATGTTGGATATTGGGTT } \\
\text { R: GCTGTCAACGATACGCTACCTA }\end{array}$ \\
\hline hsa-miR-152-3p & $\begin{array}{l}\text { F: TCAGTGCATGACAGAACTTGG } \\
\text { R: GCTGTCAACGATACGCTACCTA }\end{array}$ \\
\hline hsa-let-7c-5p & $\begin{array}{l}\text { F: TGAGGTAGTAGGTTGTATGGTT } \\
\text { R: GCTGTCAACGATACGCTACCTA }\end{array}$ \\
\hline hsa-miR-196b-5p & $\begin{array}{l}\text { F: TAGGTAGTTTCCTGTTGTTGGG } \\
\text { R: GCTGTCAACGATACGCTACCTA }\end{array}$ \\
\hline $\begin{array}{l}\text { hsa-miR-107 } \\
\text { hsa-miR-517a-3p }\end{array}$ & $\begin{array}{l}\text { F: ATCGTGCATCCCTITAGAGTGT } \\
\text { R: GCTGTCAACGATACGCTACCTA }\end{array}$ \\
\hline hsa-miR-517b-3p & $\begin{array}{l}\text { F: ATCGTGCATCCCTITAGAGTGT } \\
\text { R: GCTGTCAACGATACGCTACCTA }\end{array}$ \\
\hline hsa-miR-1307-5p & $\begin{array}{l}\text { F: TCGACCGGACCTCGACCGGCT } \\
\text { R: GCTGTCAACGATACGCTACCTA }\end{array}$ \\
\hline $18 \mathrm{~S}$ rRNA & $\begin{array}{l}\text { F: CAGCCACCCGAGATTGAGCA } \\
\text { R: TAGTAGCGACGGGCGGTGTG }\end{array}$ \\
\hline CLTA & $\begin{array}{l}\text { F: TCCAACAGACAGTTATGCAGC } \\
\text { R: CCATTTACGGATACTITCAGGCT }\end{array}$ \\
\hline CLTC & $\begin{array}{l}\text { F: ATTCTGCCAATTCGTTTCAGGA } \\
\text { R: GCTTTCAGTGCAATTACTTTGCT }\end{array}$ \\
\hline RAB3A & $\begin{array}{l}\text { F: GAGTCCTCGGATCAGAACTTCG } \\
\text { R: TGTCGTTGCGATAGATGGTCT }\end{array}$ \\
\hline SNAP25 & $\begin{array}{l}\text { F: TCGTGTAGTGGACGAACGG } \\
\text { R: TCTCATTGCCCATATCCAGGG }\end{array}$ \\
\hline SYT1 & F: GTGAGCGAGAGTCACCATGAG \\
\hline
\end{tabular}

Table 1 qPCR primers (Continued)

\begin{tabular}{|c|c|}
\hline Gene Name & $\begin{array}{l}\text { Forward (F) and reverse (R) primers } \\
\left(5^{\prime} \rightarrow 3^{\prime}\right)\end{array}$ \\
\hline & R: CCCACGGTGGCAATGGAAT \\
\hline VAMP2 & $\begin{array}{l}\text { F: CTCAAGCGCAAATACTGGTGG } \\
\text { R: TGATGGCGCAAATCACTCCC }\end{array}$ \\
\hline AP2B1 & $\begin{array}{l}\text { F: ATCCAATGGTGGTGGCTAATG } \\
\text { R: TGGGTTCAGATCAAGTAAGTTGC }\end{array}$ \\
\hline ARF6 & $\begin{array}{l}\text { F: GGGAAGGTGCTATCCAAAATCTT } \\
\text { R: CACATCCCATACGTTGAACTTGA }\end{array}$ \\
\hline ASAP3 & $\begin{array}{l}\text { F: ACGCCCAGCACAACTITITC } \\
\text { R: GGCTCAGGTGTTCCTCTCT }\end{array}$ \\
\hline DAB2 & $\begin{array}{l}\text { F: GTAGAAACAAGTGCAACCAATGG } \\
\text { R: GCCTTTAACCTTGCTAAGAGA }\end{array}$ \\
\hline PSD3 & $\begin{array}{l}\text { F: GGAGAAAGCTAACGGAACACA } \\
\text { R: TGAGGAATGTCCAAAAATGGGTT }\end{array}$ \\
\hline RAB11FIP1 & $\begin{array}{l}\text { F: AGAACAGCGAGTACGGATCTT } \\
\text { R: GACATCATTCTTAGACCGAAGGC }\end{array}$ \\
\hline STAM & $\begin{array}{l}\text { F: AGGAGCATGTGTATCAAACTGTG } \\
\text { R: TGTCCATTCAACCATAAGAGCC }\end{array}$ \\
\hline TRAF6 & $\begin{array}{l}\text { F: TTTGCTCTTATGGATTGTCCCC } \\
\text { R: CATTGATGCAGCACAGTTGTC }\end{array}$ \\
\hline
\end{tabular}

\section{RNA-Seq}

Construction of miRNA libraries and RNA-Seq was completed by KangChen Bio-tech (Shanghai, China). The haemorrhoidal tissues of 3 patients were used as the sample group and subcutaneous scar tissue of healthy people wasused as the normal control group. The miRNA libraries were constructed following the manufacturer's protocol. Briefly, the total RNA was extracted from samples in each group to prepare the miRNA sequencing libraries. This includes the following steps: 1) 3' adapter ligation; 2) 5' adapter ligation; 3) cDNA synthesis; 4) PCR amplification; 5) Selection of 135-155 bp PCR amplicons (correspond to 15-35 nt small RNAs). Libraries were denatured into single-strand DNA molecules and captured using the Illumina flow cell. This was followed by in situ amplification into clusters. Further, 51 sequencing cycles were carried out on anIllumina NextSeq 500 sequencer, following the manufacturer's instructions.

\section{Data analysis and statistical methods}

Solexa CHASTITY was used for quality control and screening of raw reads from data obtained from RNASeq to obtain clean reads. Adapters were removed from clean reads to obtain trimmed reads with a tag of $\geq 15 \mathrm{nt}$. The miRDeep2 software was used for prediction of new miRNAs from all trimmed reads to obtain new premiRNAs. Following that, the Novoalign software (v2.07.11) was used to align trimmed reads to the merged pre-miRNA database (miRBase v21 pre-miRNAs + newly predicted pre-miRNAs) with at most 1 
mismatch allowed. During calculation of miRNA expression, reads with a quantity less than 2 were discarded. In order to characterise changes in isoforms, \pm 4 nt reads in mature forms were considered as isoforms of mature miRNAs and classified as 5P and 3P species according to their position on the precursor hairpin. The number of reads in every mature miRNA region $( \pm 4 \mathrm{nt})$ was summed as the raw expression level and tag counts per million miRNA alignments (TPM) was used for standardizing samples. We calculated the expression levels of mature miRNAs, isoforms with the highest expression level, and all miRNA isoforms to satisfy the different requirements of customers. The number of tags on all standardised miRNA isoforms was used to calculate the $P$-value and fold change of the two groups of samples (with replicates) and these values were used to screen for differentially expressed miRNAs. The fold change between two samples (without replicates) was calculated to screen for differentially expressed miRNAs. A cluster map of miRNAs was plotted. A miRNA target gene prediction software was used to predict the target genes of top 10 differentially expressed miRNAs and these target genes were used for Gene Ontology (GO) and Pathway analysis.

\section{Results}

Significant differences in miRNA expression exist between haemorrhoidal tissue and healthy tissue

Before transcriptome sequencing of miRNAs was carried out, we carried out pathological staining of all collected tissue samples to determine their pathological characteristics and staging. From the H\&E staining results, several tortuous and dilated veins couldbe observedat the layer below the haemorrhoidal mucosa, with thick walls surrounding elastic fibres. Intraluminal thrombi were present in individual tissues. Interstitial oedema was apparent and diffuse linear and wavy elastic fibres were observed between collagen fibres. Many dilated and distended veins were present in the mucosa of haemorrhoids with small amounts of erythrocytes and inflammatory cells (Fig. 1). Compared with the pathological characteristics of haemorrhoidal tissue, no thrombus,

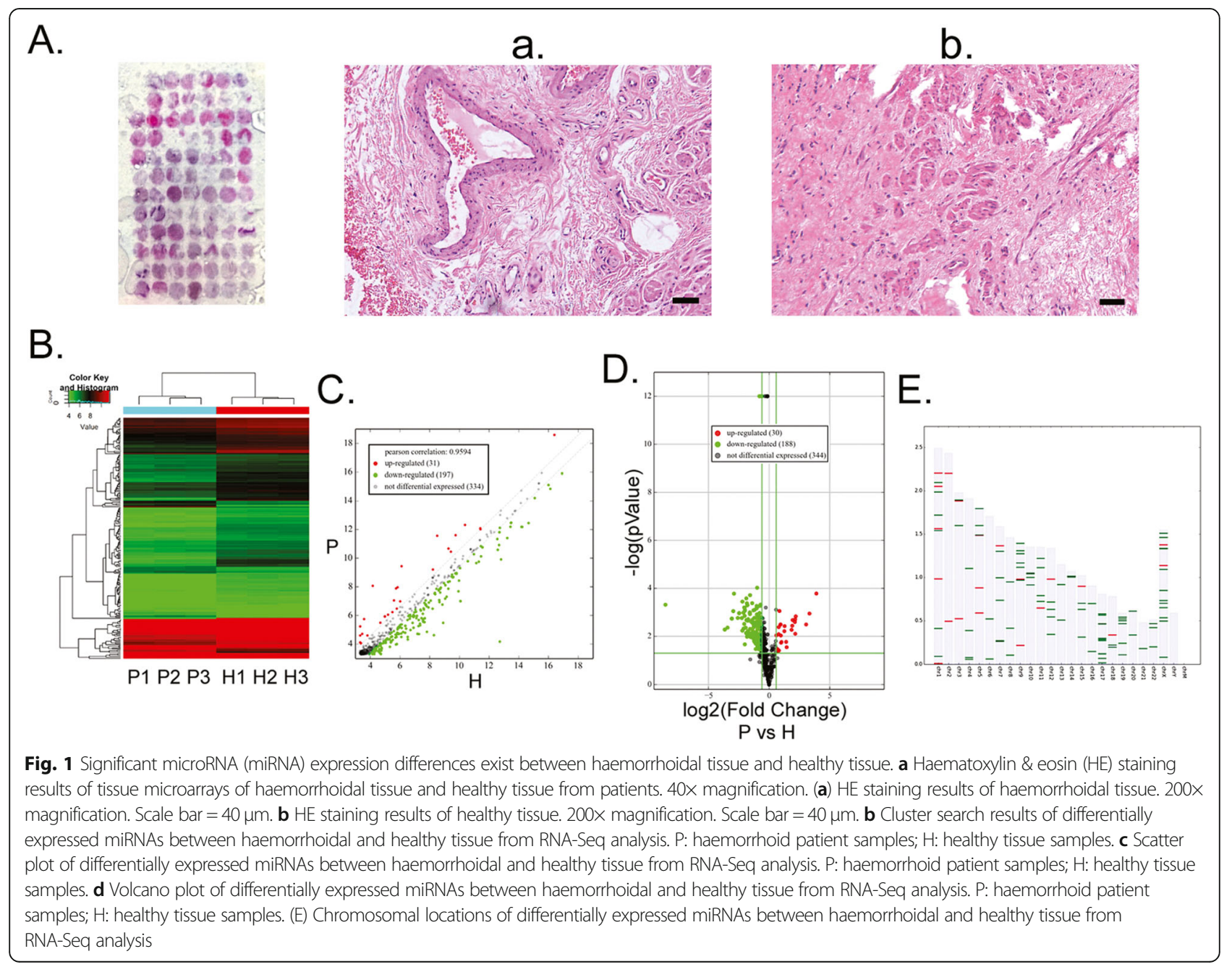


oedema, or inflammatory cells were observed in healthy tissue (Fig. 1). The reads obtained from RNA-Seq were aligned to pre-miRNAs to calculate the number of miRNA alignments as the expression level of miRNA. The miRNA expression information of mature miRNA, most abundant isomiRNA, and all isoforms were calculated and TPM (number of reads of aligned miRNA *1 million/aligned reads) was used for standardisation. Finally, all miRNA isoform data was used to calculate the differentially expressed miRNAs between samples. We obtained differential miRNA transcriptome data between haemorrhoidal and healthy tissues that are more complete and comprehensive through bioinformatics analysis. These results showed that 1942 miRNA molecules were obtained from sequencing (Fig. 1). These miRNAs are distributed in chromosomes other than the Y chromosome (Fig. 1). In these miRNAs, 9 miRNAs were significantly upregulated (ratio $>3.5$ and $P$-value $<$ 0.01 ) and 16 miRNAs were significantly downregulated (ratio $>0.6$ and $P$-value $<0.01$ ) (Table 2). The results showed that many differentially expressed miRNAs were present between haemorrhoidal and healthy tissues.
Differentially expressed miRNAs in haemorrhoids have many potential target genes

miRNAs are the most abundant class of small RNAs in mammals. They can induce mRNA degradation or inhibit transcription to play important roles in regulating gene expression. Each miRNA binds to the 3' UTR of target genes by partial sequence complementation. On average, each miRNA has a few hundred target genes. After obtaining the differentially expressed miRNAs from screening, authoritative miRNA databases (TargetScan7.1 and MirdbV5) were used for alignment. Biostatistical calculations were used to screen for potential target genes of the aforementioned miRNAs to construct miRNA-target genes regulatory networks. This was used to deduce the function of these miRNAs. In this study, we predicted the target genes of 7 significantly upregulated miRNAs and 8 significantly downregulated miRNAs (Fig. 2). Bioinformatics analysis showed that the quantities of corresponding target genes of the aforementioned miRNAs differed in different databases. For upregulated miRNAs, 502 and 908 potential target genes were screened from the TargetScan7.1 and MirdbV5

Table 2 Restults of microRNA RAN-Seq

\begin{tabular}{|c|c|c|c|c|c|c|}
\hline Access Number & microRNA & Mature Microrna Length & Mature microRNA Sequence & P vs H Fold Change & $\boldsymbol{P}$-value & State \\
\hline MIMAT0000728 & miR-375 & 22 & UUUGUUCGUUCGGCUCGCGUGA & 14.944 & $<0.000$ & up \\
\hline MIMAT0000272 & miR-215-5p & 21 & AUGACCUAUGAAUUGACAGAC & 10.072 & 0.001 & up \\
\hline MIMAT0000222 & miR-192-5p & 21 & CUGACCUAUGAAUUGACAGCC & 8.330 & 0.003 & up \\
\hline MIMAT0000435 & miR-143-3p & 21 & UGAGAUGAAGCACUGUAGCUC & 4.600 & 0.001 & up \\
\hline MIMAT0000262 & miR-187-3p & 22 & UCGUGUCUUGUGUUGCAGCCGG & 4.472 & 0.008 & up \\
\hline MIMAT0000460 & miR-194-5p & 22 & UGUAACAGCAACUCCAUGUGGA & 4.450 & 0.002 & up \\
\hline MIMAT0000437 & miR-145-5p & 23 & GUCCAGUUUUCCCAGGAAUCCCU & 3.852 & 0.002 & up \\
\hline MIMAT0002806 & miR-490-3p & 22 & CAACCUGGAGGACUCCAUGCUG & 3.767 & 0.004 & up \\
\hline MIMAT0004601 & miR-145-3p & 22 & GGAUUCCUGGAAAUACUGUUCU & 3.625 & 0.005 & up \\
\hline MIMAT0002172 & miR-376b-3p & 22 & AUCAUAGAGGAAAAUCCAUGUU & 0.667 & $<0.000$ & down \\
\hline MIMAT0000255 & miR-34a-5p & 22 & UGGCAGUGUCUUAGCUGGUUGU & 0.663 & 0.002 & down \\
\hline MIMAT0000438 & miR-152-3p & 21 & UCAGUGCAUGACAGAACUUGG & 0.657 & 0.003 & down \\
\hline MIMAT0000064 & let-7c-5p & 22 & UGAGGUAGUAGGUUGUAUGGUU & 0.657 & 0.007 & down \\
\hline MIMAT0000104 & miR-107 & 23 & AGCAGCAUUGUACAGGGCUAUCA & 0.655 & 0.008 & down \\
\hline MIMAT0002852 & miR-517a-3p & 22 & AUCGUGCAUCCCUUUAGAGUGU & 0.652 & 0.004 & down \\
\hline MIMAT0002857 & miR-517b-3p & 22 & AUCGUGCAUCCCUUUAGAGUGU & 0.652 & 0.004 & down \\
\hline MIMAT0022727 & miR-1307-5p & 21 & UCGACCGGACCUCGACCGGCU & 0.650 & 0.007 & down \\
\hline MIMAT0000458 & miR-190a-5p & 22 & UGAUAUGUUUGAUAUAUUAGGU & 0.648 & 0.004 & down \\
\hline MIMAT0000731 & miR-378a-5p & 22 & CUCCUGACUCCAGGUCCUGUGU & 0.645 & 0.000 & down \\
\hline MIMAT0004927 & miR-708-3p & 22 & CAACUAGACUGUGAGCUUCUAG & 0.635 & 0.006 & down \\
\hline MIMAT0001545 & miR-450a-5p & 22 & UUUUGCGAUGUGUUCCUAAUAU & 0.632 & 0.002 & down \\
\hline MIMAT0001545 & miR-450a-5p & 22 & UUUUGCGAUGUGUUCCUAAUAU & 0.623 & 0.006 & down \\
\hline MIMAT0000692 & miR-30e-5p & 22 & UGUAAACAUCCUUGACUGGAAG & 0.617 & 0.008 & down \\
\hline MIMAT0002888 & miR-532-5p & 22 & CAUGCCUUGAGUGUAGGACCGU & 0.616 & 0.000 & down \\
\hline
\end{tabular}




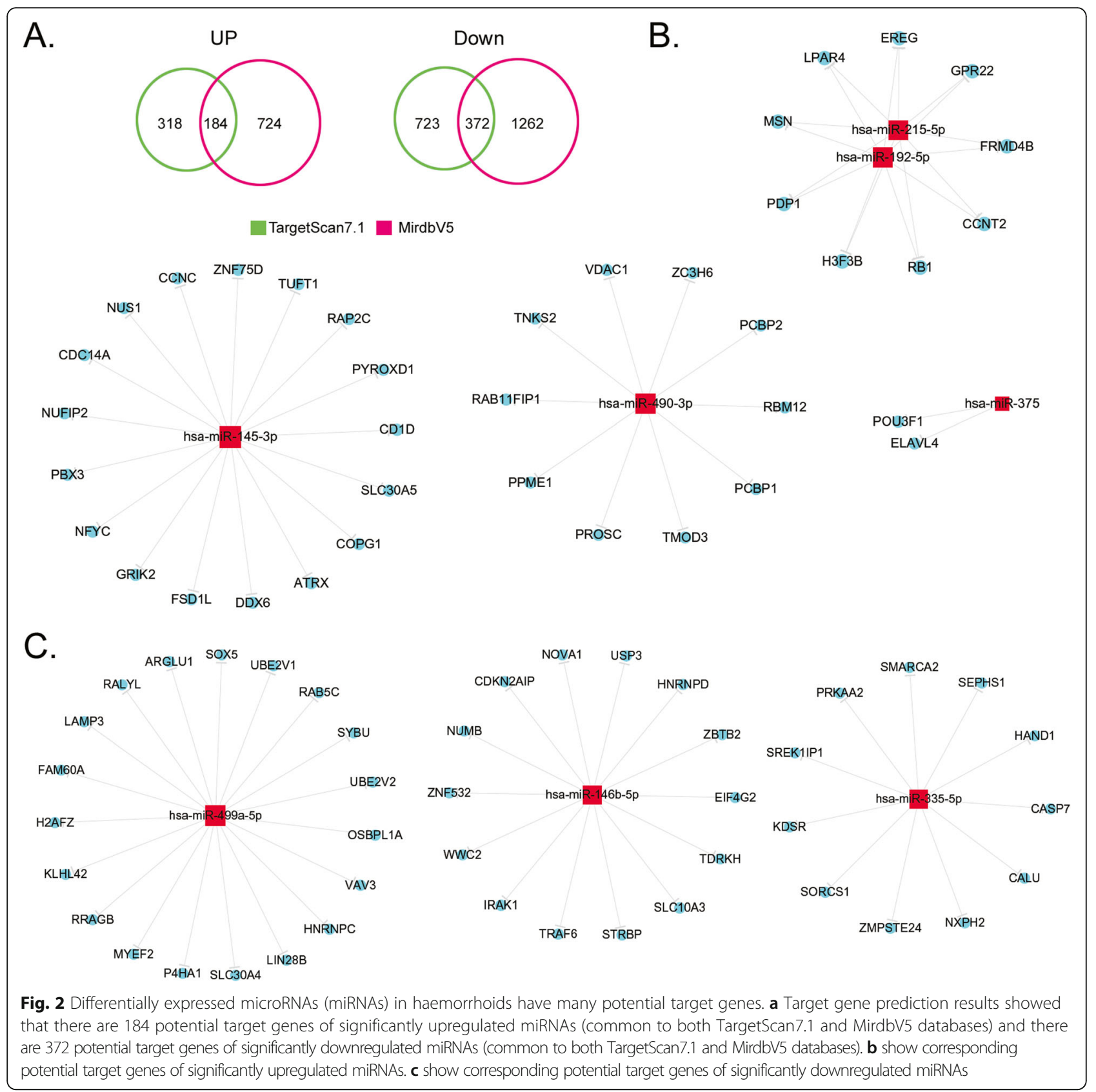

databases, respectively, of which 184 target genes were common to both databases (Fig. 2, Supplementary data). For downregulated miRNAs, 1095 and 1634 potential target genes were screened from the TargetScan7.1 and MirdbV5 databases, respectively, of which 372 target genes were common to both databases (Fig. 2, Supplementary data). We noticed that except for miRNA-133b and miRNA-133a-3p, which had 32 common potential target gene mRNAs, the predicted potential target genes of the remaining 13 miRNAs were all independent genes and there was no overlap (Fig. 2, Supplementary data).
The experimental results showed that the aforementioned miRNAs with significant differential expression regulates a large number of potential target genes.

Gene ontology analysis shows that haemorrhoids contain many physiological and biochemical abnormalities

GO is an international standard classification standard for gene function and is divided into molecular function (MF), biological process (BP), and cellular component (CC). We employed GO to analyse and deduce the molecular functions of the target genes of the 10 miRNAs 
with the most significant differential expression to predict the function of these differentially expressed miRNAs. The experimental results showed that the target genes of upregulated miRNAs are mainly involved in BP, CC, and MF (Fig. 3, Supplementary data). The target genes of downregulated miRNAs were mainly involved in BP, CC, and MF (Fig. 3, Supplementary data). GO analysis results showed that the target genes of differentially expressed miRNAs in haemorrhoids mainly regulate cell composition and protein binding.

\section{Endocytosis and synaptic vesicle cycle are possible regulatory targets of miRNAs during the development of haemorrhoids}

Through RNA-Seq, we screened out differentially expressed miRNAs between haemorrhoidal and healthy

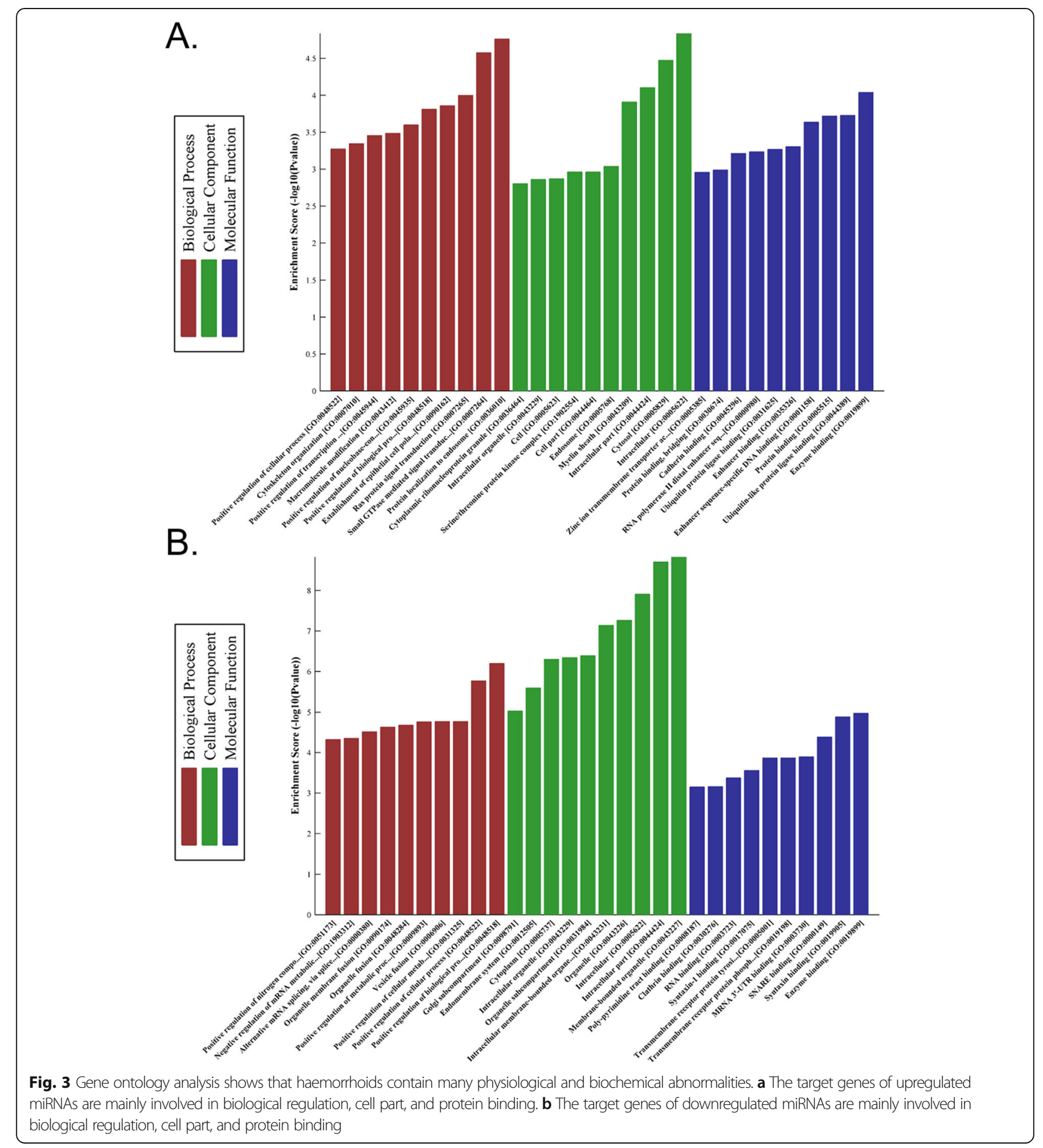


tissues. We obtained several potential target genes that are regulated by these miRNAs through alignment with target gene databases. The target genes obtained were aligned with the KEGG database (http://www.genome.jp/ $\mathrm{kegg} /$ ) to identify the biological signal transduction pathways wherethe genes are located. The alignment data showed that the gene populations that are regulated by upregulated miRNAs belong to 10 signalling pathways, of which the endocytosis pathway contained the greatest number of differentially expressed target genes (Fig. 4, Supplementary data). On the contrary, the gene populations that are regulated by downregulated miRNAs belong to 10 signalling pathways, of which the synaptic vesicle cycle pathway is the pathway that contained the greatest number of differentially expressed target genes (Fig. 5, Supplementary data). Meanwhile, the expression levels of important node genes on endocytosis or synaptic vesicle cycle signalling pathway were validated by qPCR. The results showed that the expressioon levels of all important node genes (AP2B1, ARF6, ASAP3, DAB2, PSD3, RAB11FIP1, STAM, TRAF6) on endocytosis pathways from haemorrhoid patient samples were higher significantly than them from healthy tissue samples (Fig. 4).
However, the results showed that the expression levels of all important node genes (CLTA, CLTC, RAB3A, SNAP25, SYT1, VAMP2) on Synaptic vesicle cycle pathways from haemorrhoid patient samples were lower significantly than them from healthy tissue samples (Fig. 4). Therefore, the above alignment data shows that the development of haemorrhoids may be associated with imbalances in target genes in the endocytosis and synaptic vesicle cycle pathways that are regulated by miRNAs.

\section{RNA-Seq high-throughput sequencing results are generally consistent with $\mathrm{qPCR}$ validation results}

Through data screening, we found that 24 miRNAs showed significant transcript differences between the two groups. From this, we can see that the development of haemorrhoids and differential miRNA expression showed a continuum. From further miRNA-specific qPCR validation, we found that only 1 upregulated miRNA (miR-192-5p) did not match the RNA-Seq results, while the remaining miRNAs matched the RNASeq results. For downregulated miRNAs, only 1 upregulated miRNA (miR-517-5p) matched the RNA-Seq results, while the remaining miRNAs did not match the

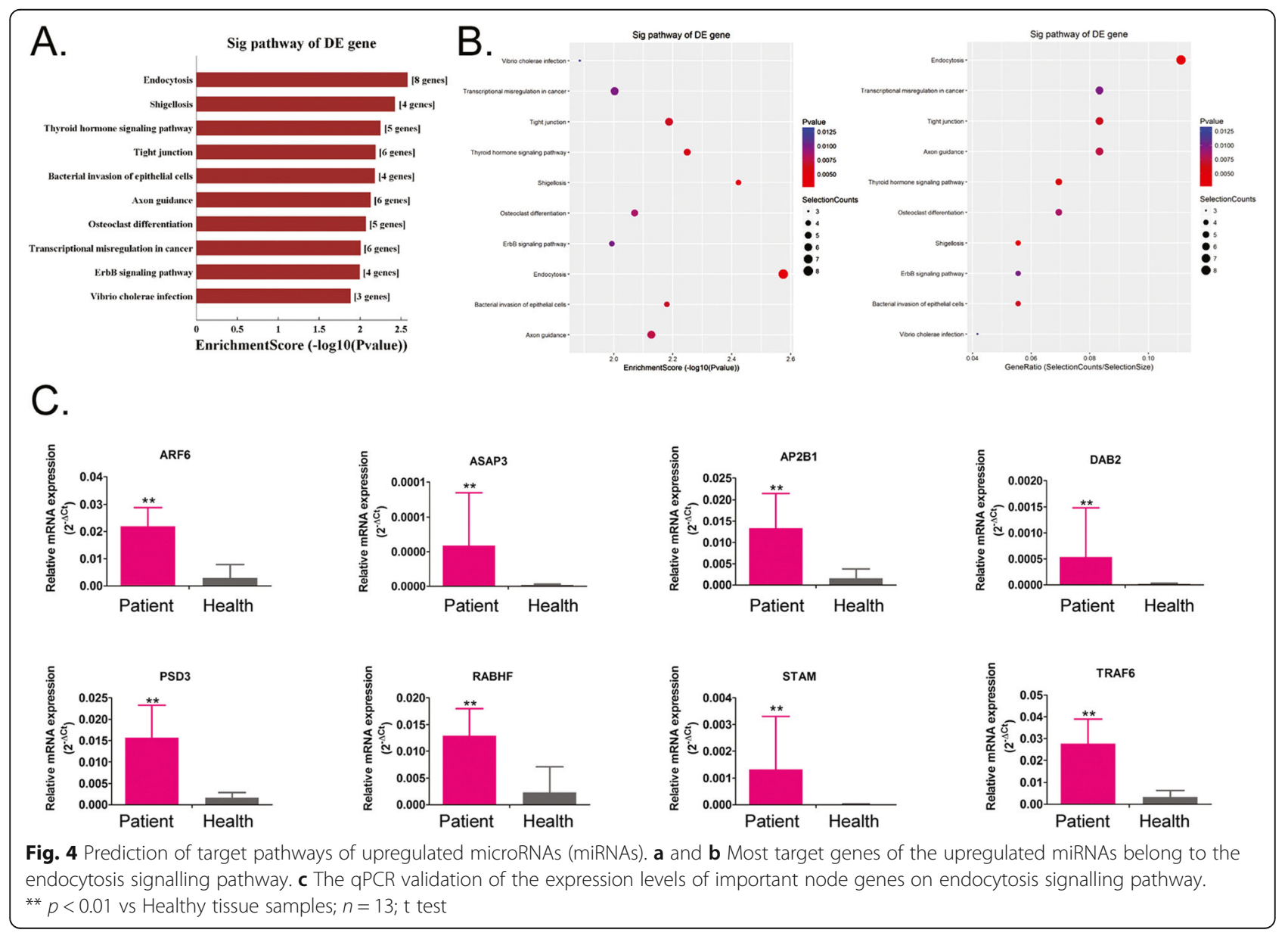




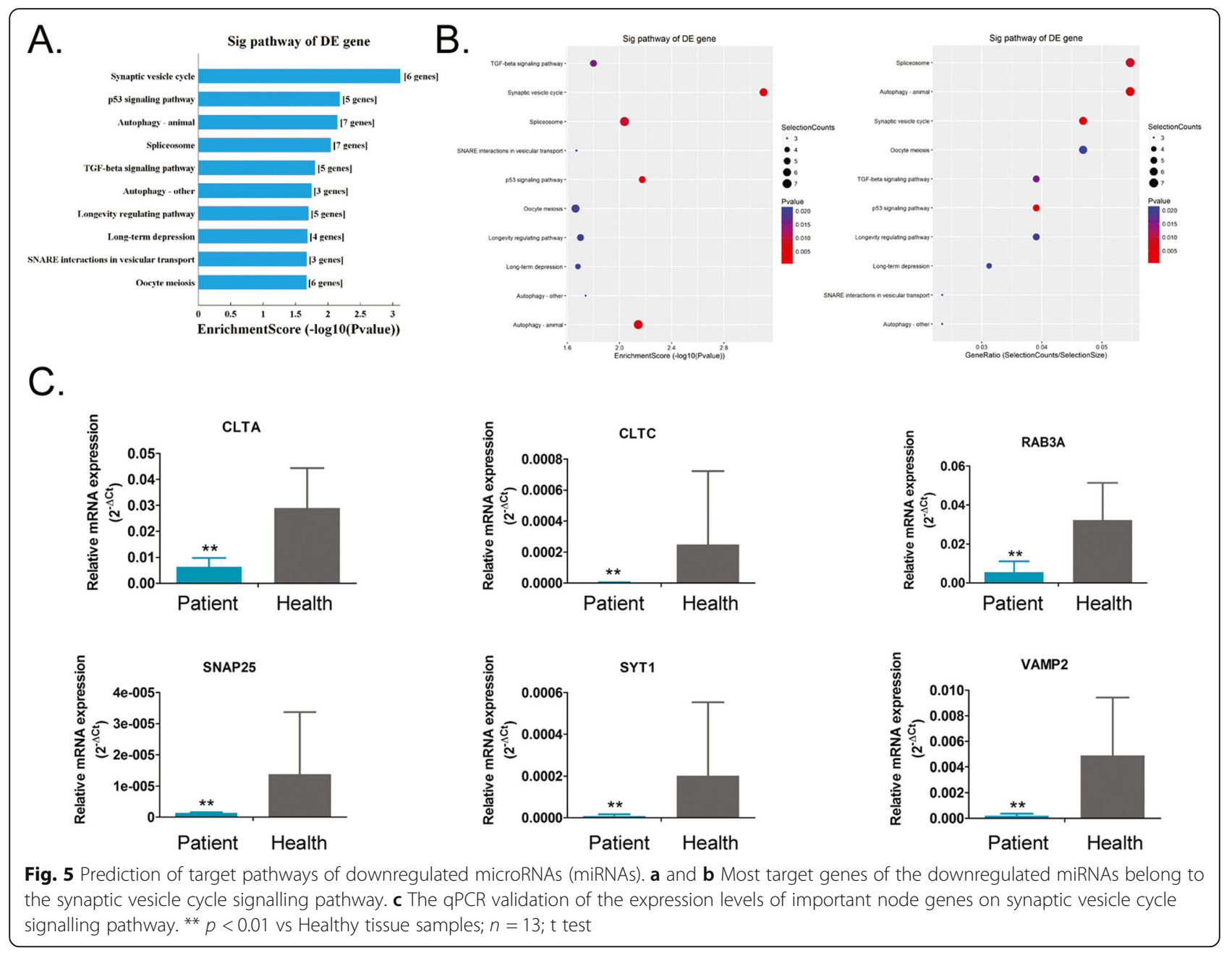

RNA-Seq results (Fig. 6). Therefore, we hypothesised that RNA-Seq has unique advantages in highthroughput screening of differentially expressed transcripts, but there are some errors with the results obtained.

\section{Discussion}

In China, haemorrhoids are a common disease in proctology $[1-6,14]$. Haemorrhoids cause much inconvenience in the lives of afflicted people and significantly affect physical and mental health. Generally, internal haemorrhoids can be classified into 4 grades $[1-6,14]$ --Grade I: Bleeding during bowel movements which spontaneously stop after bowel movement. These haemorrhoids do not prolapse; Grade II: Frequent haematochezia, protrudes out during bowel movement but spontaneously return to the original position; Grade III: Require manual pushing after prolapse; and Grade IV: Permanently prolapsed and cannot be pushed back into position [1-6, 14]. Among these haemorrhoids, Grade II and above haemorrhoids mostly form mixed haemorrhoids, which show both symptoms of internal and external haemorrhoids. Pain and itching may be present. Itching is often caused by viscous secretions during haemorrhoid prolapse [1-6, 14]. The aetiology of haemorrhoids is extremely complex. Currently, there are two relatively recognised theories. The first theory is the varicose vein theory which asserts that haemorrhoids are a mass of veins formed from the congestion, dilatation, and flexion of the venous plexus at the lower mucosa of the rectum and below the skin of the anal canal. In addition, Thomson's sliding anal canal lining theory has become more and more accepted $[1-6,14]$. This theory asserts that haemorrhoids are originally normal anatomical structures in the anal canal, i.e., vascular cushions, which are ring-like spongy tissues at the pectinate line and $1.5 \mathrm{~cm}$ above the pectinate line [1-6, 14]. Only when abnormalities in the anal cushion occur along with comorbid symptoms can they be termed as haemorrhoids and require treatment. The objective of treatment is to relieve symptoms and not to eliminate haemorrhoids $[1$, $3-6,14]$. There are many causes of haemorrhoids, such 


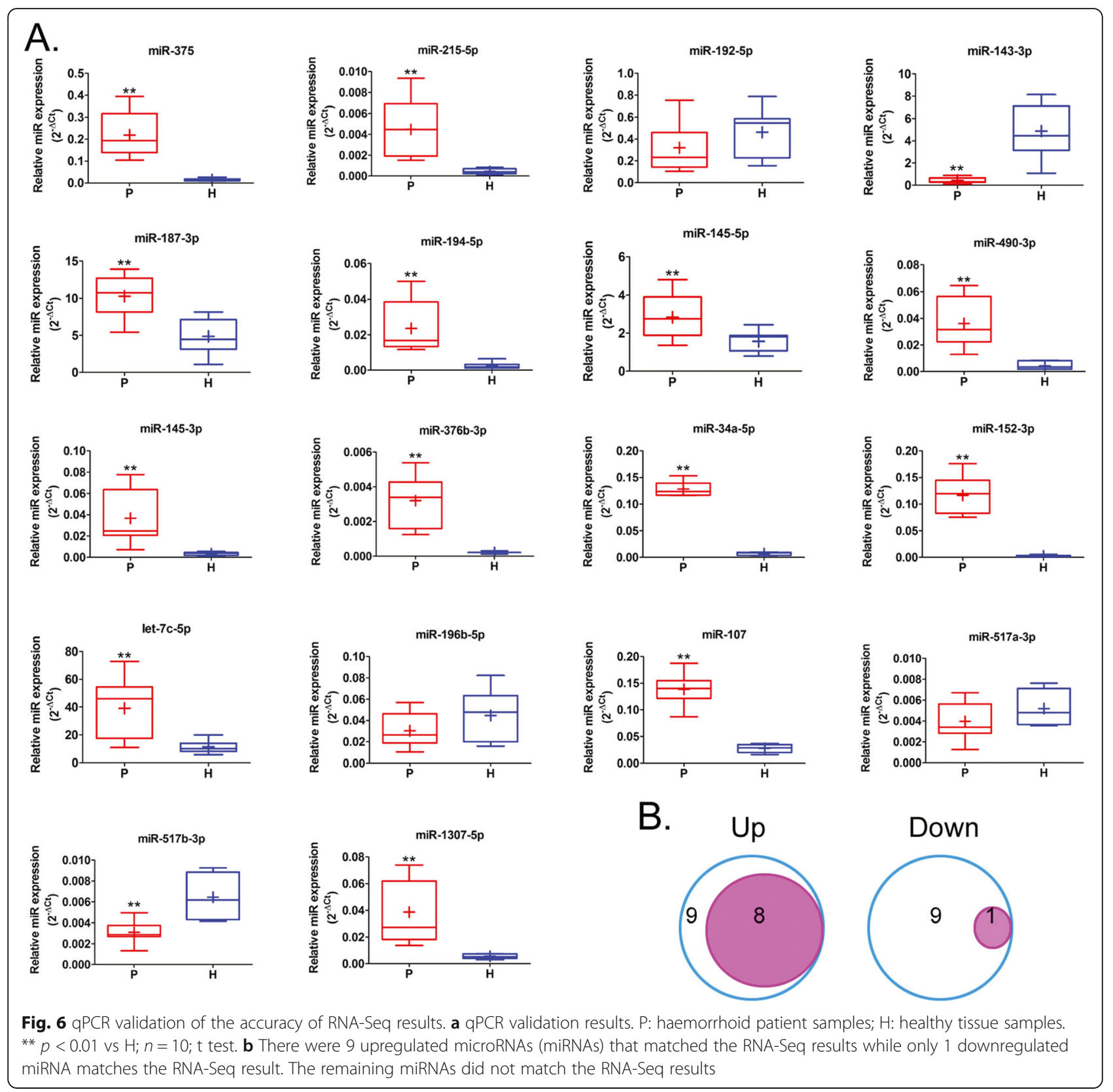

as constipation, long-term alcohol consumption, constipation of large amounts of food irritants and prolonged sitting are major causes [1-6, 14]. However, the development of haemorrhoids is considered to be intimately associated with lifestyle habits and environmental factors $[1-6,14]$. On the contrary, many reports foundthat environmental factors and lifestyle habits can significantly affect epigenetic status and induce diseases. Based on the aforementioned clues, we hypothesised that the development of haemorrhoids should have unique epigenetic variation patterns. There are not many studies in the field of epigenetic regulation on the regulatory mechanisms of non-coding RNAs, particularly miRNAs, on the development of haemorrhoids.

In our study, we focused on miRNAs. By employing RNA-Seq, we conducted a comprehensive and in-depth analysis of the differences in miRNA transcriptomics between haemorrhoidal and healthy tissues and the status of potential target genes that were regulated by these miRNAs. The RNA-Seq results showed that there are transcript differences in thousands of miRNAs between haemorrhoidal and healthy tissues, and data screening showed that 24 of these miRNAs were significantly differentially expressed between the two groups. From this, 
we can see that the development of haemorrhoids and differential miRNA expression shows a continuum. From further miRNA-specific qPCR validation, we found that only 1 upregulated miRNA did not match the RNA-Seq results, while the remaining miRNAs matched the RNASeq results. For downregulated miRNAs, only 1 upregulated miRNA matched the RNA-Seq results while the remaining miRNAs did not match the RNA-Seq results. Therefore, we hypothesised that although RNA-Seq has unique advantages in high-throughput screening of differentially expressed transcripts, there are some errors inthe results obtained. There is still a need for large numbers of clinical samples for qPCR validation of the differential transcriptome data obtained from RNA-Seq in order to identify data that are consistent with the results and remove contradictory data. Subsequently, we identified potential target genes of differentially expressed miRNAs from 2 public databases. The statistical analysis showed that there were several potential target genes, regardless of whether the miRNA was upregulated or downregulated. In addition, there are overlaps in the target genes that are regulated by some miRNAs. These results suggest that the development of haemorrhoids is due to a large and complex miRNAgene regulatory network. Changes in the expression of some miRNAs will change the expression of a group of target genes. By further classification of the potent target genes that are regulated by the miRNAs obtained from screening, we found that the endocytosis and synaptic vesicle cycle signal transduction pathways are signalling pathways that contain the most target genes. Therefore, we hypothesised that the development of haemorrhoids may be associated with imbalances in target genes in the endocytosis and synaptic vesicle cycle pathways that are regulated by miRNAs.

Aberrant responses to physiological or nonphysiological stress signals by the body are usually the cause of disease. The occurrence of haemorrhoids is a good example. This is because there are many causes of haemorrhoids, such as constipation, long-term alcohol consumption, consumptionof large amounts of food irritants, and prolonged sitting. These factors can result in long-term and frequent stimulation of the perianal and rectal regions and be considered as a stable and persistent external stress signal. The occurrence of haemorrhoids is an abnormal response of the body to these stress signals. In recent years, many studies have pointed out the body's responses to external stress signals are regulated by miRNAs and aberrant miRNA regulation are intimately associated with the occurrence of the aforementioned conditions. The rapid responses of cells towards multiple stimuli in a complex environment are achieved and completed by signal transduction pathways. At the same time, miRNAs are used to achieve regulation $[8,11]$. There are diverse miRNAs and they have many important physiological, biochemical, and gene regulatory functions. Each miRNA has many target genes and some miRNAs can regulate the same target gene. This results in the formation of a complex and intertwined signal regulatory network. One miRNA can regulate the expression of many target genes or the combination of several miRNAs can be used to fine-tune the regulation of some target genes $[8,11]$. When stress occurs, miRNAs will use the following potential mechanisms for regulation: stress signal mediation, stress signal modulation, negative feedback and signal resolution, positive feedback and phenotypic switching, and buffering and signal stability. The activation of these 5 mechanisms differs but they have a common point in responses to stress signals, i.e., requiring corresponding miRNAs to achieve responses $[8,11,15]$. Once stress occurs, the corresponding miRNAs will be activated which will directly or indirectly regulate downstream signalling pathways to achieve responses to that stress signal [15]. A study has found that a deficiency in the corresponding miRNA molecule will result in an extremely slow response to that stress signal [15].

On the other hand, Endocytosis is a process in which extracellular substances are transported into cells through the deformation of plasma membranes [16-20]. According to the different size of the materials and the different mechanisms of endocytosis, it can be divided into three types, such as phagocytosis, pinocytosis and receptor mediated endocytosis [16-20]. Besides, synaptic vesicle cycle signal transduction pathway is a signal pathway involved in the process of chemical substance transmission in neuronal signal transduction [16-18, 21-25]. The vesicular vamp protein binds SNARE protein Syntaxin 1 and Snap25 on the neuron cell membranes, which leads the vesicle to fuse [16-18, 21-25]. The Munc18-1 protein combines monomeric Syntaxin 1 and SNARE complex and assists in the assembly of the complex [16-18, 21-25]. Complexin and Syt1 bind to SNARE protein to form a tight complex, which can aggregate lipid membranes. When the action potential in presynaptic neurons opens voltage-gated calcium channels, calcium ions combine with Syt1 and interact with SNARE complex and plasma membrane to fuse the membrane and release the electrical signals of neurons to the synaptic space $[16-18,21-25]$. Above two signaling pathways cover intercellular material and signal transmission, which play an important role in cell survival, growth and normal physiological function. However, in the process of hemorrhoids, whether above signaling pathways regulate the infiltration of inflammatory cells, proliferation of vascular endothelial cells, and edema of interstitial cells and other abnormal pathophysiological processes remains to be further studied. 


\section{Conclusions}

In conclusion, this study indicates that the occurrence of haemorrhoids may be intimately associated with aberrant miRNA transcription, resulting in aberrant target gene expression and an imbalance in certain signal transduction pathways. In the future, functional studies will be conducted to define the relationship and underlying mechanism of these findings.

\section{Supplementary information}

Supplementary information accompanies this paper at https://doi.org/10. 1186/s41065-020-00139-9.

Additional file 1: Figure S1. Gene ontology analysis shows that haemorrhoids contain many physiological and biochemical abnormalities (A-F) The target genes of upregulated miRNAs are mainly involved in biological regulation (BP), cell part (CC), and protein binding (MF). (G-L) The target genes of downregulated miRNAs are mainly involved in biological regulation (BP), cell part (CC), and protein binding (MF). Figure S2. Differentially expressed microRNAs (miRNAs) in haemorrhoids have many potential target genes. (A) Target gene prediction results showed that there are 184 potential target genes of significantly upregulated miRNAs (common to both TargetScan7.1 and MirdbV5 databases) and there are 372 potential target genes of significantly downregulated miRNAs (common to both TargetScan7.1 and MirdbV5 databases). (B) and (C) show corresponding potential target genes of significantly upregulated miRNAs. (D) and (E) show corresponding potential target genes of significantly downregulated miRNAs. Figure S3. Endocytosis signalling pathway. Figure S4. Synaptic vesicle cycle signalling pathway.

\section{Abbreviations}

miRNA: microRNA; GO: Gene ontology; H\&E: Haematoxylin-eosin; TPM: Tag counts per million miRNA alignments; MF: Molecular function; BP: Biological process; CC: Cellular component

\section{Acknowledgements}

We would like to thank the native English speaking scientists of AMERICAN JOURNAL EXPERTS for editing our manuscript.

\section{Authors' contributions}

Chengkun Song, Haikun Zhou, Hong Lu, and Chunsheng Luo completed most of the experiments in the study; Te Liu, Chen Wang and Qingming Wang completed induction, collation and analysis of experimental data; Yunhua Peng completed collection of experimental samples; Te Liu, Wei Yang and Yaojie Xin completed study design, manuscript writing and provided experimental funding support. The authors read and approved the final manuscript.

\section{Funding}

This work was supported by grant from the National Natural Science Foundation of China (No. 81673768, 81574033, 81973899). And, grant from Shanghai Natural Science Foundation (No. 16401971600). And, grant from Shanghai Municipal Health Bureau Fund (No. SHDC12014222). And, grant from Shanghai University of TCM GaoFengGaoYuan Foundation (2505). And, grant from Shanghai University of TCM Foundation (2016YSN44).

\section{Ethics approval and consent to participate}

All procedures performed in studies involving human participants were in accordance with the ethical standards of Shuguang Hospital, Shanghai University of Traditional Chinese Medicine (Permission No.: 201701.4) committee and with the 1964 Helsinki declaration and its later amendments or comparable ethical standards.

\section{Consent for publication}

Not applicable.

\section{Competing interests}

The authors declare that they have no conflicts of interest.

\section{Author details}

${ }^{1}$ Department of Anorectal Surgery, Shuguang Hospital, Shanghai University of Traditional Chinese Medicine, 528 Zhangheng Road, Shanghai 201203, P.R.

China. ${ }^{2}$ Department of Otolaryngology, Shuguang Hospital, Shanghai University of Traditional Chinese Medicine, Shanghai 201203, China.

${ }^{3}$ Shanghai Geriatric Institute of Chinese Medicine, Shanghai University of Traditional Chinese Medicine, 365 South Xiangyang Road, Shanghai 200031, P.R. China. ${ }^{4}$ Department of Pathology, Yale University School of Medicine, New Haven, CT 06520, USA.

Received: 2 June 2020 Accepted: 30 June 2020

Published online: 03 July 2020

\section{References}

1. Sugerman DT. JAMA patient page, hemorrhoids. JAMA. 2014;312(24):2698.

2. Jacobs D. Clinical practice. Hemorrhoids. N Engl J Med. 2014;371(10):944-51.

3. Qureshi WA. Office management of hemorrhoids. Am J Gastroenterol. 2018; 113(6):795.

4. Fox A, Tietze PH, Ramakrishnan K. Anorectal conditions: hemorrhoids. FP Essent. 2014;419:11-9.

5. Lohsiriwat V. Treatment of hemorrhoids: a coloproctologist's view. World J Gastroenterol. 2015;21(31):9245-52.

6. Mott T, Latimer K, Edwards C. Hemorrhoids: diagnosis and treatment options. Am Fam Physician. 2018;97(3):172-9.

7. Lu J, Getz G, Miska EA, Alvarez-Saavedra E, Lamb J, Peck D, et al. MicroRNA expression profiles classify human cancers. Nature. 2005;435(7043):834-8.

8. Leung AK, Sharp PA. MicroRNA functions in stress responses. Mol Cell. 2010; 40(2):205-15.

9. van Rooij E. The art of microRNA research. Circ Res. 2011;108(2):219-34.

10. Borghini A, Andreassi MG. Genetic polymorphisms offer insight into the causal role of microRNA in coronary artery disease. Atherosclerosis. 2018; 269:63-70.

11. Creugny A, Fender A, Pfeffer S. Regulation of primary microRNA processing. FEBS Lett. 2018:592(12):1980-96.

12. Mishra P, Singh D, Ganju L, Kumar B. MicroRNA in gastrointestinal cell signalling. Inflammopharmacology. 2018;26(1):1-14.

13. Wang C, Lu H, Luo C, Song C, Wang Q, Peng Y, et al. Yang W: miR-412-5p targets Xpo1 to regulate angiogenesis in hemorrhoid tissue. Gene. 2019;705:167-76.

14. Rakinic J, Poola VP. Hemorrhoids and fistulas: new solutions to old problems. Curr Probl Surg. 2014;51(3):98-137.

15. Mendell JT, Olson EN. MicroRNAs in stress signaling and human disease. Cell. 2012;148(6):1172-87.

16. Gan Q, Watanabe S. Synaptic vesicle endocytosis in different model systems. Front Cell Neurosci. 2018;12:171.

17. Saheki Y, De Camilli P. Synaptic vesicle endocytosis. Cold Spring Harb Perspect Biol. 2012;4(9):a005645.

18. Yamashita T. Ca2+-dependent regulation of synaptic vesicle endocytosis. Neurosci Res. 2012;73(1):1-7.

19. Birgisdottir $A B$, Johansen T. Autophagy and endocytosis - interconnections and interdependencies. J Cell Sci. 2020;133:10.

20. Joseph JG, Liu AP. Mechanical regulation of endocytosis: new insights and recent advances. Adv Biosyst. 2020;4(5):e1900278.

21. Blakely RD, Edwards RH. Vesicular and plasma membrane transporters for neurotransmitters. Cold Spring Harb Perspect Biol. 2012;4:2.

22. Jahn R, Fasshauer D. Molecular machines governing exocytosis of synaptic vesicles. Nature. 2012;490(7419):201-7.

23. Miyano R, Miki T, Sakaba T. Ca-dependence of synaptic vesicle exocytosis and endocytosis at the hippocampal mossy fibre terminal. J Physiol. 2019; 597(16):4373-86.

24. Daniel JA, Malladi CS, Kettle E, McCluskey A, Robinson PJ. Analysis of synaptic vesicle endocytosis in synaptosomes by high-content screening. Nat Protoc. 2012:7(8):1439-55.

25. Sudhof TC. A molecular machine for neurotransmitter release: synaptotagmin and beyond. Nat Med. 2013;19(10):1227-31.

\section{Publisher's Note}

Springer Nature remains neutral with regard to jurisdictional claims in published maps and institutional affiliations. 